\title{
Child Security Enhancement System using Raspberry Pi and Amazon Elasticsearch Service
}

\author{
Anjo A N J, Vinay M
}

\begin{abstract}
According to recent surveys child missing, child kidnapping cases are rapidly increasing in India [1]. Child's safety in school buses and outside school premises is one of main concern for the parents today. The proposed system tries to provide security features to the children using new methodologies that are added to the existing safety system for better protection. The proposed system consists of portable unit, cloud system and android application. The portable unit consists of raspberry pi 2 model B, GPS receiver with antenna and pulse rate sensor. This unit will track the position of the child in the form of latitude longitude and altitude using GPS receiver and sense the pulse rate values using heart beat sensor. These data are sending to raspberry pi module which is then injected into elasticsearch with the help of internet connectivity. In cloud system elastic search which is served by amazon cloud service provides a URL which extracts the indexed values from the data injected by raspberry pi device. The android application in a user interface that shows the location of the child in map, path in which the child travelled and the speed in which child moves. The pulse rate of the child is also monitored in application real-time. The URL produced by the elasticsearch is used to retrieve values to the application.

Keywords-Raspberry Pi 2 model B, GPS receiver, pulse rate sensor, elasticsearch, Amazon web service
\end{abstract}

\section{INTRODUCTION}

Large number of children travel to the school every day in India. Children go in different modes of transportation. Parents are always considered about the safety and security of their child. This paper intends to provide location and health tracking system which ensures the safety of the school going children. Parents can monitor the child's location and health in real time so that they can ensure that their child is safe. In some cases kids forget to leave or enter school bus or even miss the bus from school.

The proposed system will helps the user to know the location of the child in case of any missing happens. This system will provide guidance for the user where they can track the location of the child and monitor the speed, distance; pulse rate of the child, as well as it shows the path travelled by the child. In case of emergency situations parent receives notifications from the system through an application and can send alert information to the concern authorities. Android developer studio is one of the best tool or environment to create android applications to mobile [2]. This software is used to develop mobile application which accesses all the information from amazon cloud server. Accessing and manipulating information from a remote application is made possible with the help of cloud computing. In the proposed system Amazon cloud is used as

Revised Manuscript Received on April 12, 2019.

Anjo A N J, BCA, st philomena's college mysore, Karnataka, India.

Vinay M, Banglore, Karnataka, India. cloud server, it receives the formatted data from Raspberry Pi. Amazon elastic search service is used to do index mapping on data, with the help of elasticsearch service and real-time application monitoring is made possible.

Python 2.7 is used to program hardware module in the system. Presently python is one of the most top listed and efficient software and hardware programming tool. Python program is used to retrieve geographical positioning values to find the child's location, which are taken out from NMEA values that are carried through GPS receiver [3]. This entire mathematical transformation process is achieved using python 2.7. Raspberry pi2 is a one board hardware device that helps students and researchers to explore computing. The main computing element of the proposed system is Raspberry pi 2 model B. The main task of Raspberry pi is to capture unformatted data from GPS receiver, process these raw data into latitude, longitude, altitude and send it to amazon web service using python code and elastic search technique.

\section{PROBLEM STATEMENT}

Presently, the current technologies for child security system are by using RFID chips, GSM modules and GPS devices which work for identifying the presence of child in school and in school bus. This kind of system does not provide any security features other than the location of the child .Most of the user interface will be in the form of website or will connected to an external monitoring system which is not user friendly. Suppose consider the child is in dangerous circumstances the existing system produce position of the child only on certain conditions. The focus of this paper is to develop a system which provides complete security to the child which is monitored real-time and is reported to the application which is used by parent. Elastic search technique is used to transfer data between different modules in the system for quick results.

\section{LITERATURE REVIEW}

An IOT based system [4] that provides security to the women. System consist of GPS that sends signs about area to raspberry pi and allows to send alert messages about the location details of the women to the predefined phone numbers. Other security feature like capturing images of the attackers and working of auto defense system with button press is implemented in this paper. 


\section{Child Security Enhancement System Using Raspberry Pi And Amazon \\ Elasticsearch Service}

The work [5] is a tracking system to the vehicles that uses GPS devices to automatically gather the information like current location, these data are transmitted using GSM devices and stored in server maintained using cloud infrastructure .Client web portal is used to access the data from the cloud server. Only the authorized users can access the data from the server. The user registration is done using vehicle identification number.

This [6] system collects the geographical coordinates using child's mobile device and send them to the guardian's application that then displays the movement of the child in Google map. The mobile device of the child detects the abnormal behavior like playing in the road; the system sends voice remainder to the child telling to stay off the road. The transfer of information between the child and the guardian is happening via internet.

In this system [7] RFID tags used to track the presence of child in school bus. It detects the entering and exiting of the children and identifies each child with the information stored in RFID tag. Name of each child is displayed in monitor that helps the driver to know whether the child is inside the bus or not. An additional feature of speed monitoring system is also implemented in the school bus, in case of over speed it alerts the driver to reduce the speed for the safety of the children. Along with RFID tags, GPS and GSM modules are used to track the location of the bus

This system [8] tries to ensure the safety of the women with the help of electronic jacket. This jacket consist of Raspberry Pi, GPS, GSM, shock circuit camera and a buzzer .this paper is mainly focusing on security of the women during odd hours or unsafe condition. The system consists of three buttons and each button carries certain functionalities, like sending the current location to saved phone numbers, switching on the alarm system and activating the shock circuit. The camera in the system will capture the images and is saved to the memory card in the system.

This is a tracking device [9] which is installed in a passenger bag. This device will track the location, if the bag is lost or stolen.it tracks the location of the bag and send the location details to the owner's android application. This system uses Arduino board, GSM and GPS modules which are placed inside the bag send the position details to the owner. It uses Thinkspeak cloud API's to transfer data between the device and the application

\section{PROPOSED WORK}

This proposed work is cloud based child security system using android platform and elastic search technique has been developed.it helps the users by tracking the information of the child using efficient technologies without any third party involvement. For instance, consider the child has gone missing then to find the location of the child ,a particular procedure has to be followed, that is lodge a complaint to an authorized people to trace the child which involves a third party. But in this system the parents can trace the location of child. It can also monitor path, speed and the pulse rate of the child. As discussed earlier the security of the child is ensured using the systems like raspberry, GPS module and elastic search technique and the application is developed using android studio. There are several concepts play here which work together to produce an efficient system, which is capable of providing safety for the children

\section{Raspberry pi 2 model b}

Raspberry pi is a microcomputer in which multiple tasks can be performed. It uses Raspbian as operating system which is Linux based [10]. Python script is inbuilt in raspberry pi for python programming. It also supports programming languages like $\mathrm{c} / \mathrm{c}++$. In this system the programming language used is python. Raspberry pi is the main component in this system. it takes the data from the GPS receiver serially. The pulse data from the pulse sensor is also provided to it for monitoring pulse rate of the child .all these data are then sent to amazon cloud elastic search service

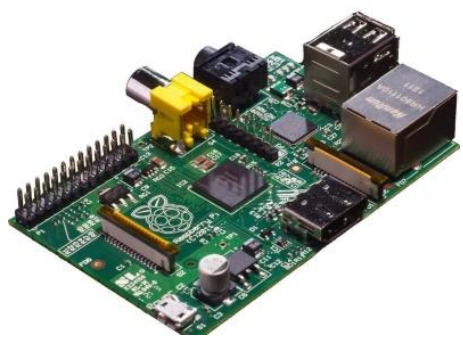

Fig1.Raspberry Pi 2 model B

\section{Pulse sensor}

Pulse sensor is a device that is used to detect the heart rate of a person. This device can be useful for students, athlete, artists, who want to monitor their live heart rate data. The sensor clips to any part of the body and plug it into any microcontroller to detect heart rate it uses operating voltage of range $3.3 \mathrm{v}-5 \mathrm{v}$ and $4 \mathrm{~mA}$ current[11] .Pulse rate sensor detects the heartbeat of the child and send it to raspberry pi using analog to digital converter.

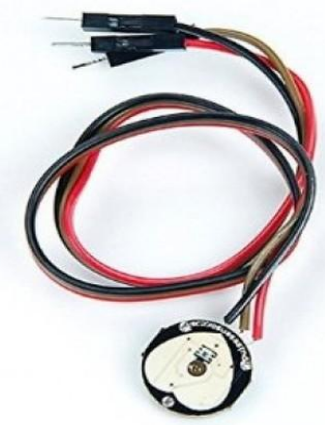

Fig.2 Pulse sensor

\section{GPS Module}

MT 5212 GPS module is used to receive GPS data from satellite. Using this module the received data can be showed in any terminal [12] in this system the received GPS values are send to raspberry pi using serial wires. 


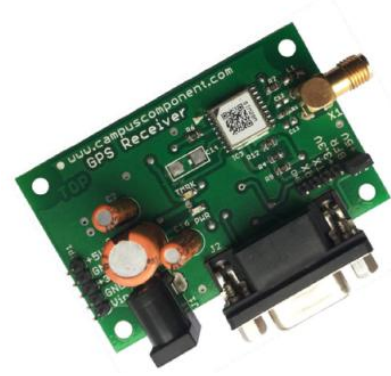

Fig.3 GPS Receiver

\section{Analog to digital converter}

Mcp3008 is a cheap 8-channel 10 bit analog to digital converter [13]. This chip can be used to read simple analog signals like from light or pulse sensor to the raspberry pi.in this system it uses mcp3008 analog to digital converter chip to read the pulse rate in raspberry pi

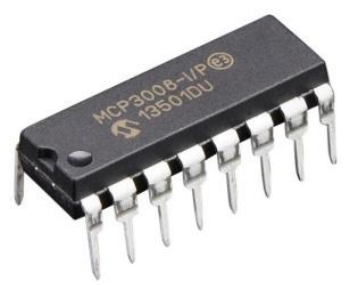

Fig.4 Mcp3008

\section{Python 2.7}

In this system the hardware module programmed using python 2.7[14]. The latitude, longitude, altitude and pulse rate values to view the child's location and heart beat rate which are received from GPS receiver and pulse sensor are conveyed through raspberry $\mathrm{Pi}$. This whole process conversion of GPS values and transportation of both GPS and pulse rate values are done by python $2.7[15]$

\section{Amazon web service (AWS)}

Amazon Web service (AWS) is a computational cloud environment developed by Amazon.com. Web service application can act as both remote computational provider as well as cloud service provider. Amazon Elastic Compute Cloud (EC2) provides a reliable and scalable cloud computing platform [16]. The specified instance type act as hardware component for the host computer. Each instance type provides different compute, storage abilities and is categorized into instance groups based on their capabilities. This system uses

The system uses Amazon web service EC2 instance t2.micro as cloud server in the proposed system.

\section{Elasticsearch}

Elasticsearch service is a managed search method to create, operate and utilize elastic search clusters in amazon cloud.

Elasticsearch is a popular free search and analysis engine used for monitoring real-time applications. Using Amazon elastic search service we can directly access the elasticsearch API'S
The data coming from raspberry pi is directly injected to the elasticsearch using a unique URL provided by amazon cloud [17]. In elasticserach an index should be created were the raspberry pi data is referred.so whenever the raspberry pi sends the data it goes through the index created in elasticsearch service. Data will be temporarily stored in JSON format [18].

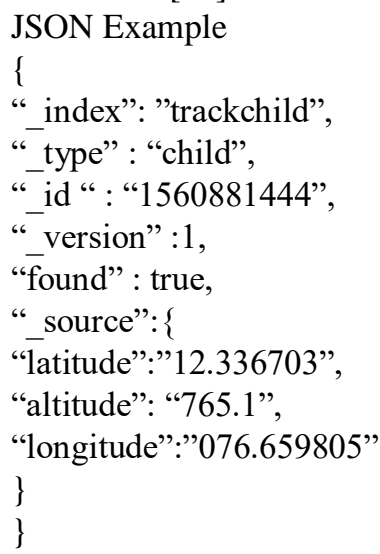

These JSON data can be extracted to other applications using the URL along with the index name provided.

\section{RESULT}

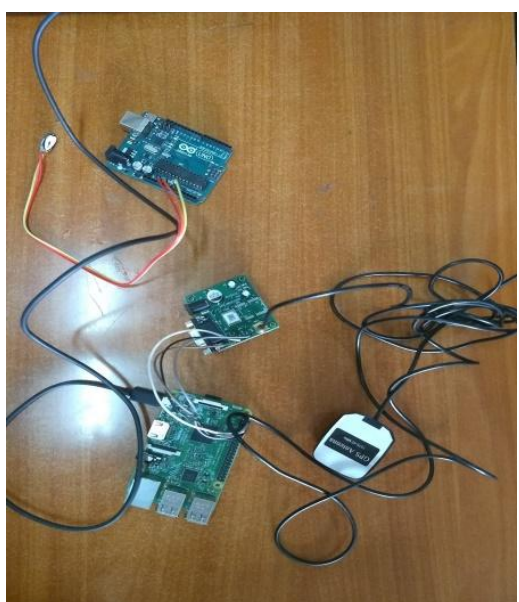

Fig.5 Hardware setup of child security system

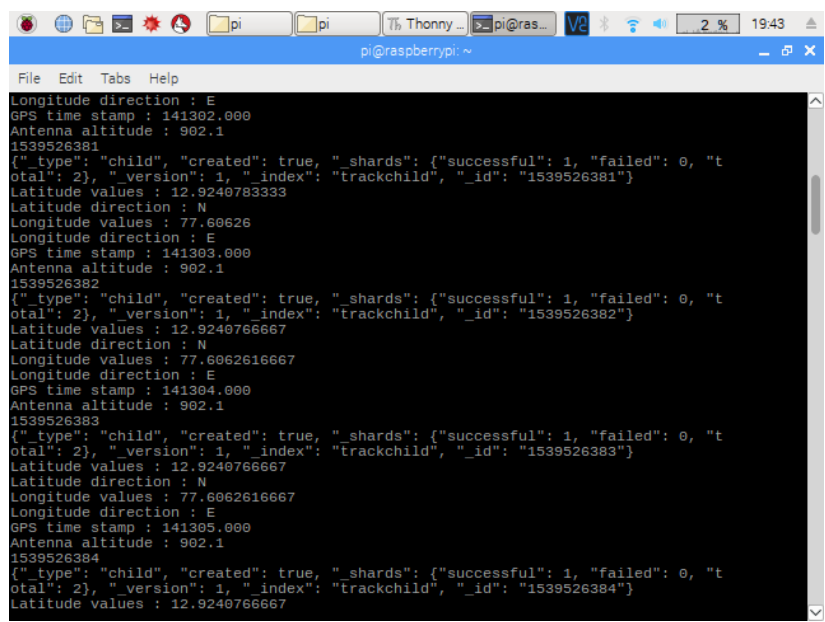

Fig.6 Reading from GPS receiver and sending to elasticsearch 


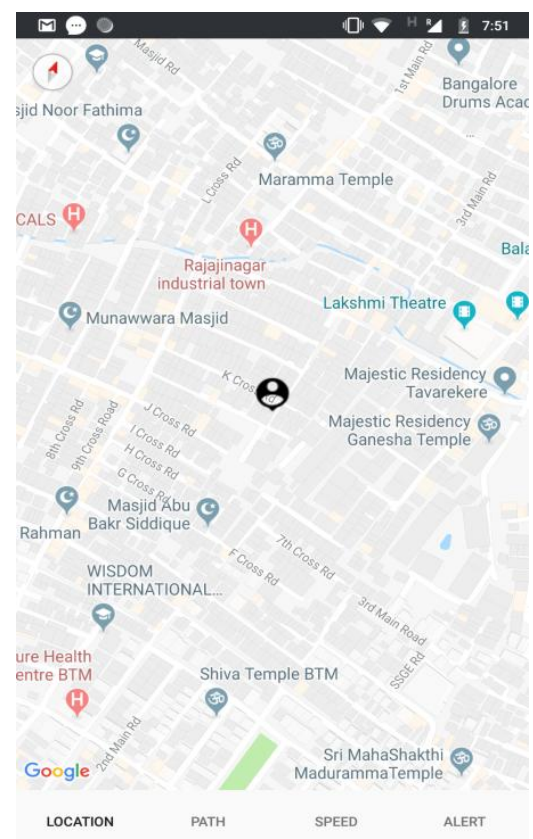

Fig.7 Location screen

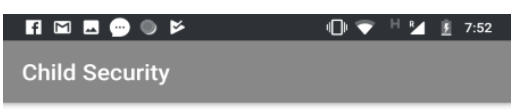

CURRENT SPEED $(\mathrm{Km} / \mathrm{Hr})$

43.

HIGHEST SPEED $(\mathrm{Km} / \mathrm{Hr})$

67.0

ALTITUDE(M)

9017

Fig.9 speed screen

\section{Location activity}

This is a user interface screen to the parent to find the location of the child. This activity will display the current position of the child in real-time. The position changes according to the movements of the child .This activity will help the parent to easily locate the child's location.

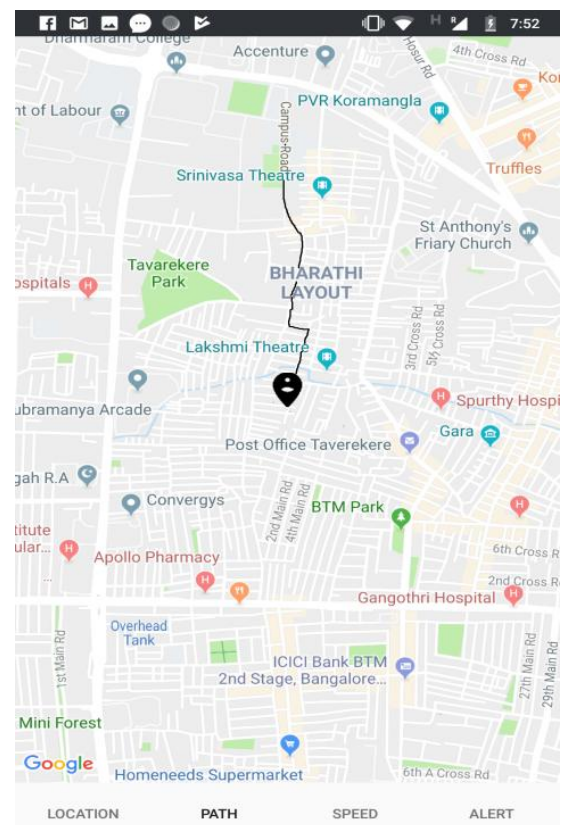

Fig.8 Path screen

\section{Path activity}

User interface screen to show the paths in which the child travelled. It shows the path from the time the system is on still the system gets off. It draws a line in the map showing the path travelled. This helps the parent to know the movements of the child in there phone.

\section{Speed activity}

This interface will display the current speed of the child and the highest speed the child travelled. It also displays the altitude of the child's location. This helps the parents to know the speed details of their child and will alert the parent if the speed exceeds the limit.

\section{CONCLUSION AND FUTURE SCOPE}

This system will continuously monitor the child and provide real time data to the parent with the help of amazon elastic search. Parents can use android application to track the live movements and also help to check the health condition of the child with this system. Elastic search methodology will help to get more accurate values and helps for quick transfer of data among real-time applications without the use of large about of memory space. Child's health condition is monitored with the help of pulse sensor and a health report about the child is notified into parent's mobile phone. The system can be further extended by adding camera for capturing the accidental conditions and sending it to the parent's phone using this efficient methodology. An alarm system can also be implemented in this system were the alarm will be set to on during dangerous situation and emergency call can be set to the parent's phone and to nearby police station. So this proposed system is providing security features to the children using new methodologies were parents can track them and ensure their safety.

\section{REFERENCES}

1. Missing children in india. (2016). [online] Available at: http://www.childlineindia.org.in/missing-children-india.html [Accessed 8 Oct. 2016].

2. Download Android Studio and SDK tools | Android Developers. (n.d.). Retrieved from https://developer.android.com/studio/ 
3. DePriest.D.(n.d.).Retrievedfrom.https://www.gpsinformation. org/dale/nmea.htm

4. Sune, R. S., \& Nerkar, M. H. (2018). Internet of Things Based Women Tracking and Security with Auto-Defender System. International Journal on Recent and Innovation Trends in Computing and Communication, 6(6), 63-68.

5. Sunehra, D. (2016). Real Time Vehicle Tracking on Google Maps using Raspberry Pi Web Server. International Journal on Recent and Innovation Trends in Computing and Communication, 4(6), 325-330.

6. Permatasari, O., \& Masruroh, S. U. (2016, April). A prototype of child monitoring system using motion and authentication with Raspberry Pi. In Cyber and IT Service Management, International Conference on (pp. 1-6). IEEE.

7. Bhor, M., Kadam, N., Shinde, D. and Mane, P. (2017). Children Safety and School Bus Tracking Solution. International Journal of Electrical, Electronics and Computer Systems (IJEECS), 5(1), pp.19-22.

8. Gharge, S. (2018). Women Safety Jacket. International Journal for Research in Applied Science and Engineering Technology,6(3), 2606-2609. doi:10.22214/ijraset.2018.3421

9. Taneja, S., Karthik, M., Shukla, M., \& Sharma, H. K. (2017). Architecture of IOT based Real Time Tracking System.

10. Opensource.com. (n.d.). What is a Raspberry Pi?. [online] Available at: https://opensource.com/resources/raspberry-pi [Accessed 24 Sep. 2016].

11. World Famous Electronics 1lc. (2011). Heartbeats in Your Project, Lickety-Split . [online] Available at: https://pulsesensor.com/ [Accessed 20 Nov. 2016].

12. Electronics For You. (2014). GPS on Raspberry Pi Electronics For You. [online] Available at: https://electronicsforu.com/electronics-projects/hardwarediy/gps-raspberry-pi [Accessed 7 May 2016].

13. Learn.adafruit.com. (n.d.). MCP3008 | Raspberry Pi Analog to Digital Converters | Adafruit Learning System. [online] Available at: https://learn.adafruit.com/raspberry-pi-analogto-digital-converters/mcp3008 [Accessed 12 Oct. 2017].

14. Python.org. (2001). Welcome to Python.org. [online] Available at: https://www.python.org/ [Accessed 20 Dec. 2016].

15. Amalgjose.com. (2015). python program to read gps values | Amal G Jose. [online] Available at: https://amalgjose.com/tag/python-program-to-read-gpsvalues/ [Accessed 9 Aug. 2017].

16. Amazon Web Services, Inc. (n.d.). Amazon EC2 Instance Types - Amazon Web Services (AWS). [online] Available at: https://aws.amazon.com/ec2/instance-types/ [Accessed 3 Aug. 2017].

17. Elastic.co. (n.d.). Getting Started with Elasticsearch | Elastic. [online] Available at: https://www.elastic.co/webinars/gettingstarted-elasticsearch?elektra=home\&storm $=$ sub1 [Accessed 8 Dec. 2016].

18. Elastic.co. (n.d.). Getting Started with Elasticsearch | Elastic. [online] Available at: https://www.elastic.co/webinars/gettingstarted-elasticsearch?elektra=home\&storm $=$ sub1 [Accessed 8 Dec. 2016].

\section{AUTHORS PROFILE}

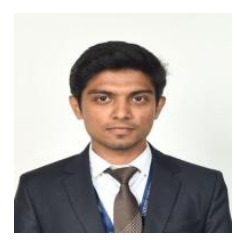

Anjo A N J completed bachelor of computer application(BCA) form st philomena's college mysore and completed master of computer application from Christ university Bangalore. Published vehicle tracking system in a journal in 2016 .Got first prize in inter college science exihibition for IOT project

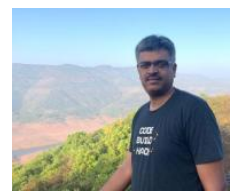

Vinay M Assistant professor Christ University bangalore published many papers on scopus indexed journals google certified educator 